\title{
A Cauchy kernel for slice regular functions
}

\author{
Fabrizio Colombo \\ Dipartimento di Matematica, Politecnico di Milano \\ Via Bonardi, 9, 20133 Milano, Italy, fabrizio.colombo@polimi.it \\ Graziano Gentili \\ Dipartimento di Matematica, Università di Firenze, \\ Viale Morgagni, 67 A, Firenze, Italy, gentili@math.unifi.it \\ Irene Sabadini \\ Dipartimento di Matematica, Politecnico di Milano \\ Via Bonardi, 9, 20133 Milano, Italy, irene.sabadini@polimi.it
}

August 10, 2008

\begin{abstract}
In this paper we show how to construct a regular, non commutative Cauchy kernel for slice regular quaternionic functions. We prove an (algebraic) representation formula for such functions, which leads to a new Cauchy formula. We find the expression of the derivatives of a regular function in terms of the powers of the Cauchy kernel, and we present several other consequent results.
\end{abstract}

AMS Classification: 30G35.

Key words: Non commutative and regular Cauchy kernel, slice regular quaternionic functions, representation formula for regular functions.

\section{Introduction}

The interest in creating a theory of quaternionic valued functions of a quaternionic variable, which would somehow resemble the classical theory of holomorphic functions of one complex variable, has produced several interesting theories. The classical, best known theory is the one due to Fueter [7], 8] (see also [6] for recent developments). In recent papers, adopting an approach used by Cullen, the authors introduced a new theory of regular functions for quaternionic and Clifford valued functions, [3], 44, [1], [12, 13]. For these new classes of functions, that will be called slice regular (resp. slice monogenic), Cauchy representation formulas were introduced in the afore mentioned papers, by means of kernels that are not slice regular (resp. not slice monogenic). Cauchy formulas and Cauchy kernels play a key role in the study of holomorphic functions and of Fueter regular functions. As in the case of holomorphic functions, Cauchy formulas are a crucial tool in the construction of functional calculus for slice regular and slice monogenic functions. In the quaternionic case, the functional calculus is associated to quaternionic operators (see for example [1] and [2]), while in the Clifford algebras case it deals with $n$-tuples of noncommuting operators (see [3], [5]). It is therefore clear that particular attention has to be put in the construction of the Cauchy kernels and the Cauchy formulas for slice regular 
functions. In the present paper, we obtain a new Cauchy formula for slice regular functions in terms of a regular Cauchy kernel, while the case of slice monogenic functions is treated in [3].

Let $\mathbb{H}$ be the real associative algebra of quaternions with respect to the basis $\{1, i, j, k\}$ satisfying the relations

$$
\begin{gathered}
i^{2}=j^{2}=k^{2}=-1, i j=-j i=k, \\
j k=-k j=i, \quad k i=-i k=j .
\end{gathered}
$$

We will write a quaternion $q$ as $q=x_{0}+i x_{1}+j x_{2}+k x_{3}\left(x_{i} \in \mathbb{R}\right)$ its conjugate as $\bar{q}=$ $x_{0}-i x_{1}-j x_{2}-k x_{3}$, and we will write $|q|^{2}=q \bar{q}$. We will also denote the real part $x_{0}$ of $q$ by $\operatorname{Re}(q)$ and its imaginary part $i x_{1}+j x_{2}+k x_{3}$ by $\operatorname{Im}(q)$. The symbol $\mathbb{S}$ will indicate the 2 -sphere of purely imaginary, unit quaternions, i.e.

$$
\mathbb{S}=\left\{q=i x_{1}+j x_{2}+k x_{3} \mid x_{1}^{2}+x_{2}^{2}+x_{3}^{2}=1\right\}
$$

and we will often use the fact that any non real quaternion $q$ can be written in a unique way as $q=x+y I$ for $x, y \in \mathbb{R}, y>0$ and $I \in \mathbb{S}$. In particular we will set

$$
I_{q}=\left\{\begin{array}{l}
\frac{\operatorname{Im}(q)}{|\operatorname{Im}(q)|} \quad \text { if } \operatorname{Im}(q) \neq 0 \\
\text { any element of } \mathbb{S} \text { otherwise }
\end{array}\right.
$$

Now, to better explain the problem we deal with, we first of all take the case of the regular functions in the sense of Fueter. Consider the the (left) Cauchy-Fueter operator

$$
\frac{\partial}{\partial \bar{q}}=\frac{\partial}{\partial x_{0}}+i \frac{\partial}{\partial x_{1}}+j \frac{\partial}{\partial x_{2}}+k \frac{\partial}{\partial x_{3}}
$$

If $U$ is an open set of $\mathbb{H}$, then a real differentiable function $f: U \rightarrow \mathbb{H}$ is called (left) Fueter regular if

$$
\frac{\partial}{\partial \bar{q}} f(q)=0
$$

for all $q \in U$. By writing the units on the right in (1), one obtains the so called right CauchyFueter operator whose kernel gives the right Fueter regular functions. The two theories of left and right Fueter regular functions are completely equivalent. The function $G(q)$ defined by

$$
G(q)=\frac{q^{-1}}{|q|^{2}}=\frac{\bar{q}}{|q|^{4}}
$$

is called the Cauchy-Fueter kernel, it is a generalization of the classical Cauchy kernel for holomorphic functions and it is used to find a Cauchy formula. In fact the function $G(q)$ turns out to be left and right Fueter regular on $\mathbb{H} \backslash\{0\}$ and we have that: if $S$ is a four dimensional domain $S \subset U$ whose boundary $\partial S$ is a compact, orientable hypersurface, and if $q_{0}$ belongs to the interior of $S$, then

$$
f\left(q_{0}\right)=\frac{1}{2 \pi^{2}} \int_{\partial S} G\left(q-q_{0}\right) D q f(q)
$$

where, with obvious notations, $D q$ is the quaternion valued 3-differential form defined by $d x_{1} \wedge$ $d x_{2} \wedge d x_{3}-i d x_{0} \wedge d x_{2} \wedge d x_{3}-j d x_{0} \wedge d x_{3} \wedge d x_{1}-k d x_{0} \wedge d x_{1} \wedge d x_{2}$.

We will now pass to the case of slice regular functions, and we will recall the definition given by the authors in [12]. 
Definition 1.1. Let $U \subseteq \mathbb{H}$ be an open set and let $f: U \rightarrow \mathbb{H}$ be a function. Let $I \in \mathbb{S}$ and let $f_{I}$ be the restriction of $f$ to the complex line $L_{I}:=\mathbb{R}+I \mathbb{R}$ passing through 1 and $I$. We say that $f$ is a left slice regular (or regular) function if, for every $I \in \mathbb{S}$

$$
\bar{\partial}_{I} f=\frac{1}{2}\left(\frac{\partial}{\partial x}+I \frac{\partial}{\partial y}\right) f_{I}(x+I y)=0
$$

and we say it is right slice regular (or right regular) if for every $I \in \mathbb{S}$

$$
f \bar{\partial}_{I}=\frac{1}{2}\left(\frac{\partial}{\partial x}+\frac{\partial}{\partial y} I\right) f_{I}(x+I y)=0
$$

We define the $I$-derivative of $f$ in $q=x+y I$ by

$$
\partial_{I} f(x+y I):=\frac{1}{2}\left(\frac{\partial}{\partial x}-I \frac{\partial}{\partial y}\right) f_{I}(x+y I)
$$

and we are now able to give the following notion of derivative:

Definition 1.2. Let $\Omega$ be a domain in $\mathbb{H}$, and let $f: \Omega \rightarrow \mathbb{H}$ be a regular function. The slice derivative (in short derivative) of $f, \partial_{s} f$, is defined as follows:

$$
\partial_{s}(f)(x+y I)=\partial_{I}(f)(x+y I) .
$$

Notice that the definition of derivative is well posed because it is applied only to regular functions for which

$$
\frac{\partial}{\partial x} f(x+y I)=-I \frac{\partial}{\partial y} f(x+y I) \quad \forall I \in \mathbb{S},
$$

and therefore, analogously to what happens in the complex case,

$$
\partial_{s}(f)(x+y I)=\partial_{I}(f)(x+y I)=\partial_{x}(f)(x+y I) .
$$

Note that if $f$ is a regular function, then its derivative is still regular because

$$
\bar{\partial}_{I}\left(\partial_{s} f(x+I y)\right)=\partial_{s}\left(\bar{\partial}_{I} f(x+I y)\right)=0,
$$

and therefore

$$
\partial_{s}^{n} f(x+y I)=\frac{\partial^{n} f}{\partial x^{n}}(x+y I) .
$$

For $R>0$, let now $B(0, R)=\{q \in \mathbb{H}:|q|<R\}$ be the open ball of radius $R$ of $\mathbb{H}$, let $f: B(0, R) \rightarrow \mathbb{H}$ be a slice regular function and let $q=x+y I_{q} \in B(0, R)$. Then the Cauchy formula for slice regular quaternionic functions states that ([12])

$$
f(q)=\frac{1}{2 \pi} \int_{\partial \Delta_{q}(0, r)}(\zeta-q)^{-1} d \zeta_{I_{q}} f(\zeta)
$$

where $d \zeta_{I_{q}}=-I_{q} d \zeta$ and $r>0$ is such that $\overline{\Delta_{q}(0, r)}:=\left\{x+I_{q} y \mid x^{2}+y^{2} \leq r^{2}\right\}$ contains $q$ and is contained in $B(0, R)$.

It is easy to prove that the function $g(\zeta)=(\zeta-q)^{-1}$ is not slice regular unless $q \in \mathbb{R}$. It is important at the same time to notice that the function $g$ is effectively used only in the complex plane $L_{I_{q}}$ which contains the point $q$. One may wonder if it is possible to consider a variation of the Cauchy formula (5) in which the corresponding kernel is regular and the path of integration does not depend on the plane $L_{I_{q}}$ to which the point $q$ belongs. 
The main results in this paper show that both questions have affirmative answers. The key tool used to obtain our results will be the function

$$
-\left(q^{2}-2 q \operatorname{Re}[s]+|s|^{2}\right)^{-1}(q-\bar{s})
$$

which turns out to be the regular inverse $(s-q)^{-*}$ of $R_{s}(q)=(s-q)$ (see [9]). The function $(s-q)^{-*}$ is, actually, the unique slice regular extension of $(s-q)^{-1}$ (in the variable $q$ ) out of $L_{I_{q}}$ and will be called the noncommutative Cauchy kernel. In particular, as a first significant step, formula (5) can be rewritten in terms of the noncommutative Cauchy kernel as

$$
f(q)=\frac{1}{2 \pi} \int_{\partial \Delta_{q}(0, r)}(\zeta-q)^{-1} d \zeta_{I_{q}} f(\zeta)=\frac{1}{2 \pi} \int_{\partial \Delta_{q}(0, r)}(\zeta-q)^{-*} d \zeta_{I_{q}} f(\zeta)
$$

where, as before, $d \zeta_{I_{q}}=-I_{q} d \zeta$ and $r>0$ is such that $\overline{\Delta_{q}(0, r)}:=\left\{x+I_{q} y \mid x^{2}+y^{2} \leq r^{2}\right\}$ contains $q$ and is contained in $B(0, R)$. The new Cauchy formula that we present in this paper holds naturally for domains - that we will call circular, slice domains - which intersect the real axis and are invariant under the action of purely imaginary rotations in $\mathbb{H}$ :

Theorem 1.3. Let $\Omega \subseteq \mathbb{H}$ be a circular, slice domain such that $\partial\left(\Omega \cap L_{I}\right)$ is union of a finite number of rectifiable Jordan arcs. Let $f$ be a regular function on $\Omega$ and, for any $I \in \mathbb{S}$, set $d s_{I}=-I d s$. Then for every $q \in \Omega$ we have:

$$
f(q)=\frac{1}{2 \pi} \int_{\partial\left(\Omega \cap L_{I}\right)}-\left(q^{2}-2 \operatorname{Re}(s) q+|s|^{2}\right)^{-1}(q-\bar{s}) d s_{I} f(s) .
$$

Moreover the value of the integral depends neither on $\Omega$ nor on the imaginary unit $I \in \mathbb{S}$.

On circular, slice domains, indeed, the proof of the Cauchy formula is achieved by means of the following representation formula, which is another crucial result obtained in the paper:

Theorem 1.4. Let $f$ be a regular function on a circular domain $\Omega \subseteq \mathbb{H}$. Choose any $J \in \mathbb{S}$. Then the following equality holds for all $q=x+y I \in \Omega$ :

$$
f(x+y I)=\frac{1}{2}[f(x+y J)+f(x-y J)]+I \frac{1}{2}[J[f(x-y J)-f(x+y J)]] .
$$

We present several other consequent results, and we find the expression of the derivatives of a regular function in terms of the powers of the regular Cauchy kernel.

\section{The Cauchy formula with regular kernel}

We will recall here the most salient properties of slice regular functions. When no confusion can arise, we will refer to left slice regular functions simply as regular functions. When it will be needed we will specify if we are considering left or right regularity.

Remark 2.1. On a domain $U \subseteq \mathbb{H}$, left regular functions form a right $\mathbb{H}$-vector space and right regular functions form a left $\mathbb{H}$-vector space. It is not true, in general, that the product or the composition of two (left/right) regular functions is (left/right) regular.

One of the key features of this notion of regularity is the fact that polynomials $\sum_{n=0}^{N} q^{n} a_{n}$ in the quaternion variable $q$, and with quaternionic coefficients $a_{n}$, are left regular (while polynomials $\sum_{n=0}^{N} a_{n} q^{n}$ are right regular). Moreover, any power series $\sum_{n=0}^{+\infty} q^{n} a_{n}$ (or more in general $\left.\sum_{n=0}^{+\infty}\left(q-p_{0}\right)^{n} a_{n}, p_{0} \in \mathbb{R}\right)$ is left regular in its domain of convergence. Conversely, every regular function on a open ball with center at the origin can be represented by a power series. Indeed we have, [12]: 
Theorem 2.2. If $B=B(0, R)$ is the open ball centered in the origin with radius $R>0$ and $f: B \rightarrow \mathbb{H}$ is a left regular function, then $f$ has a series expansion of the form

$$
f(q)=\sum_{n=0}^{+\infty} q^{n} \frac{1}{n !} \frac{\partial^{n} f}{\partial x^{n}}(0)
$$

converging on B. Analogously, if $f$ is right regular it can be expanded as

$$
f(q)=\sum_{n=0}^{+\infty} \frac{1}{n !} \frac{\partial^{n} f}{\partial x^{n}}(0) q^{n} .
$$

In both cases $f$ is infinitely differentiable on $B$

It is straightforward that an analogous statement holds for regular functions in an open ball centered in any $p_{0} \in \mathbb{R}$. Note that, even though the definition of regular function involves the direction of the unit quaternion $I$, the coefficients of the series expansion do not depend upon the choice of $I$.

A basic result in the theory of regular functions that we will need in the sequel is the following version of the identity principle (see [12]):

Theorem 2.3 (Identity Principle). Let $f: B(0, R) \rightarrow \mathbb{H}$ be a regular function. Denote by $Z_{f}=\{q \in B: f(q)=0\}$ the zero set of $f$. If there exists $I \in \mathbb{S}$ such that $L_{I} \bigcap Z_{f}$ has an accumulation point, then $f \equiv 0$ on $B$.

The following definitions can be found in [10], and are intended to overcome the difficulties arising from the facts that product of regular functions, and the conjugate of a regular function, are not regular in general.

Definition 2.4. Let $f(q)=\sum_{n=0}^{+\infty} q^{n} a_{n}$ and $g(q)=\sum_{n=0}^{+\infty} q^{n} b_{n}$ be given quaternionic power series converging on $B(0, R)$. We define the regular product of $f$ and $g$ as the series $f * g(q)=$ $\sum_{n=0}^{+\infty} q^{n} c_{n}$, where $c_{n}=\sum_{k=0}^{n} a_{k} b_{n-k}$ for all $n \in \mathbb{N}$.

The series expansion of $f * g$ converges on $B(0, R)$ (see [9]), and a similar definition can be given for right regular functions whose regular product will have the coefficients on the left. When considering polynomials in the quaternionic variable $q$ with coefficient on the left (thus right regular functions), this definition of regular product coincides with the standard multiplication of polynomials with coefficients in a noncommutative ring (see, e.g., [14]).

Definition 2.5. Let $f(q)=\sum_{n=0}^{+\infty} q^{n} a_{n}$ be a given quaternionic power series with radius of convergence $R$. We define:

- the regular conjugate of $f$ as the series $f^{c}(q)=\sum_{n=0}^{+\infty} q^{n} \bar{a}_{n}$

- the symmetrization of $f$ as $f^{s}=f * f^{c}$.

Since $f^{s}(q)=\sum_{n=0}^{+\infty} q^{n} r_{n}$, where $r_{n}=\sum_{k=0}^{n} a_{k} \bar{a}_{n-k} \in \mathbb{R}$ for all $n \in \mathbb{N}$, we have that $f^{s}=$ $f * f^{c}=f^{c} * f$ has real coefficients. An analogous definition, that we will use without stating it explicitly, holds for right regular power series.

The Cauchy kernel which we will define and study in this paper is inspired by the need to have a suitable Cauchy formula to extend the functional calculus for quaternionic operators to functions defined on more general domains. Indeed, in the complex case the kernel $(\zeta-z)^{-1}$ is 
the sum of the series $\sum_{n \geq 0} z^{n} \zeta^{-1-n}$, which is obtained by the standard series development. In the quaternionic case, the same arguments shows

$$
\begin{gathered}
(s-q)^{-1}=\left(\left(1-q s^{-1}\right) s\right)^{-1}=s^{-1}\left(1-q s^{-1}\right)^{-1} \\
=s^{-1} \sum_{n \geq 0}\left(q s^{-1}\right)^{n}=\sum_{n \geq 0} s^{-1}\left(q s^{-1}\right)^{n} .
\end{gathered}
$$

If we now fix $s=u+v I$ and take $z \in L_{I}$ with $|z|<|s|$, the previous expression can be written as

$$
(s-z)^{-1}=\sum_{n \geq 0} z^{n} s^{-1-n} .
$$

This expression is holomorphic on the disc $\Delta=\Delta(0,|s|)$ in $L_{I}$ and therefore, it can be extended uniquely by the Identity Principle to a regular function on the ball $B(0,|s|)$ in $\mathbb{H}$

$$
\sum_{n \geq 0} q^{n} s^{-1-n}
$$

This explains the meaning of the following definition (see [1], 2]):

Definition 2.6. Let $q, s \in \mathbb{H}$ such that $s q \neq q s$. We will call non commutative Cauchy kernel series (shortly Cauchy kernel series) the series expansion

$$
S^{-1}(s, q):=\sum_{n \geq 0} q^{n} s^{-1-n}
$$

for $|q|<|s|$.

Proposition 2.7. The Cauchy kernel series is left regular in $q$ and right regular in $s$, respectively, for $|q|<|s|$.

Proof. It is an immediate consequence of Theorem 2.2.

We will now show two possible approaches to the construction of a regular Cauchy kernel function. The first one is direct: we compute the Cauchy kernel function and then we show that it is regular. The second one makes use of the so called regularization process introduced in [9]. Let us start by the direct approach introduced, in a different setting, in [1], [2]. In these two papers we proved the following:

Theorem 2.8. Let $q$ and $s$ be two quaternions such that $q s \neq s q$ and consider

$$
S^{-1}(s, q):=\sum_{n \geq 0} q^{n} s^{-1-n}
$$

Then the inverse $S(s, q)$ of the quaternion $S^{-1}(s, q)$ is the nontrivial solution to the equation

$$
S^{2}+S q-s S=0
$$

Remark 2.9. An algebraic equation with quaternionic coefficients can be suitably rewritten with the coefficients on one side. When algebraic equations are written with coefficients on one side, they may have isolated zeroes or 2-dimensional spheres of solutions. In particular, a degree two equation has either two isolated zeroes or a 2 -sphere of zeroes (see e.g. [14]). Since $S=0$ is a trivial solution of (9), also the second solution must be isolated. 
Remark 2.10. Note that $R(s, q)=s-q$ is a solution of equation (9) if and only if $s q=q s$.

We have the following result:

Theorem 2.11. Let $q, s \in \mathbb{H}$ be such that $q s \neq s q$. Then the non trivial solution of

$$
S^{2}+S q-s S=0
$$

is given by

$$
\begin{gathered}
S(s, q)=(q-\bar{s})^{-1} s(q-\bar{s})-q \\
=-(q-\bar{s})^{-1}\left(q^{2}-2 q \operatorname{Re}(s)+|s|^{2}\right) .
\end{gathered}
$$

Proof. The result has been proved in [2] by directly verifying that $S(s, q)$ is a solution. Here we show how to find the solution using the techniques developed in [15], [16]. We remark that it is possible to find the same result also using the so-called Niven's algorithm method which however involves longer computations. We transform the equation $S^{2}+S q-s S=0$ into another one having coefficients on the left. Set

$$
S:=W-q
$$

and replace it in equation (10) to get

$$
(W-q)(W-q)+(W-q) q-s(W-q)=0
$$

so the equation becomes

$$
W^{2}-(s+q) W+s q=(W-s) *(W-q)=0,
$$

where $*$ denotes the left regularized product. One root is $W=q$ (see [16]), while the second root is $W=(q-\bar{s})^{-1} s(q-\bar{s})$, thus $S=(q-\bar{s})^{-1} s(q-\bar{s})-s$. By grouping $(q-\bar{s})^{-1}$ on the left we obtain (11).

Definition 2.12. The function defined by

$$
S^{-1}(s, q)=-\left(q^{2}-2 q \operatorname{Re}(s)+|s|^{2}\right)^{-1}(q-\bar{s}) .
$$

will be called Cauchy kernel function.

Note that we are using the same symbol $S^{-1}$ to denote both the Cauchy kernel series and the Cauchy kernel function. Indeed they coincide where they are both defined by virtue of their regularity (see Proposition 2.17) and in view of the Identity Principle.

Proposition 2.13. For any $q, s \in \mathbb{H}$ such that $q \neq \bar{s}$ the following identity holds:

$$
(q-\bar{s})^{-1} s(q-\bar{s})-q=(s-\bar{q}) q(s-\bar{q})^{-1}+s,
$$

or, equivalently,

$$
-(q-\bar{s})^{-1}\left(q^{2}-2 q \operatorname{Re}(s)+|s|^{2}\right)=\left(s^{2}-\operatorname{Re}(q) s+|q|^{2}\right)(s-\bar{q})^{-1} .
$$


Proof. One may prove the identities by direct computations but we prefer to follow here a shorter approach. Let us solve equation (10) by transforming it into an equation with right coefficients by setting

$$
S:=W+s
$$

and replacing it in the equation. We get

$$
(W+s)(W+s)+(W+s) q-s(W+s)=W^{2}+W(s+q)+s q=0 .
$$

This equation can be split as $(W+s) *(W+q)=0$, where $*$ denotes the (right) regular product. It is immediate, see [9], that one root is $W=-s$ while the second is $W=(-\bar{q}+s) q(-\bar{q}+s)^{-1}$. These two roots correspond to $S=0$ and $S=(s-\bar{q}) q(s-\bar{q})^{-1}+s$ which coincides with (11) when written in the form $S=\left(s^{2}-\operatorname{Re}(q) s+s^{2}\right)(s-\bar{q})^{-1}$.

As announced, let us now follow the second approach to find $S^{-1}(s, q)$. When $q, s$ commute, the sum of the Cauchy kernel series is the function $R^{-1}(q)=(s-q)^{-1}$. Since this last function is, in general, not regular, we construct its regular extension. A crucial point is that the regular extension of $R^{-1}$ coincides with the sum of the Cauchy kernel series.

Proposition 2.14. Let $f(q)$ be a regular function in $B(0, R)$. Its inverse with respect to the regular product is the function

$$
f^{-*}(q)=\left(f^{s}(q)\right)^{-1} f^{c}(q) .
$$

The function $f^{-*}$ is regular on $B(0, R) \backslash\left\{q \in \mathbb{H}: f^{s}(q)=0\right\}$.

Proof. An easy computation shows that

$$
\left(f^{-*} * f\right)(q)=\left(f^{s}(q)\right)^{-1}\left(f^{c} * f\right)(q)=1 .
$$

Moreover, the series expansion $f^{s}(q)=\sum_{n=0}^{+\infty} q^{n} r_{n}$ has real coefficients and hence $\left(f^{s}(q)\right)^{-1}$ is regular; indeed, for $I \in \mathbb{S}$, if we set $z=x+y I$ we have:

$$
\begin{gathered}
\frac{\partial}{\partial x}\left(f^{s}(x+y I)\right)^{-1}=-\left(f^{s}(x+y I)\right)^{-2} \frac{\partial}{\partial x} f^{s}(x+y I) \\
\frac{\partial}{\partial y}\left(f^{s}(x+y I)\right)^{-1}=-\left(f^{s}(x+y I)\right)^{-2} \frac{\partial}{\partial y} f^{s}(x+y I) I=-I\left(f^{s}(x+y I)\right)^{-2} \frac{\partial}{\partial y} f^{s}(x+y I) .
\end{gathered}
$$

It is immediate now to verify that $\left(\frac{\partial}{\partial x}+I \frac{\partial}{\partial y}\right)\left(f^{s}(x+y I)\right)=0$ for all $I \in \mathbb{S}$. The regularity of $f^{-*}$ follows by the regularity of $f^{c}$ and by the fact that $\left(f^{s}\right)^{-1} * f^{c}=\left(f^{s}\right)^{-1} f^{c}$ since $\left(f^{s}\right)^{-1}$ has real coefficients.

Remark 2.15. We point out that $\left(f^{s}\right)^{-1} * f^{c}(q)=\left(f^{s}\right)^{-1} f^{c}(q)=f^{c} *\left(f^{s}\right)^{-1}(q)$. If we construct a regular inverse with respect to the left regularized product, we get an analogous formula where the symbol $*$ denotes the left regularized product: $\left(f^{s}\right)^{-1} * f^{c}(q)=f^{c} *\left(f^{s}\right)^{-1}(q)=f^{c}\left(f^{s}\right)^{-1}(q)$. Note that the only difference is the position of $f^{c}$ when using the standard product.

By applying the previous proposition to $R(s, q)=s-q$ we obtain:

Proposition 2.16. The inverse with respect to the regular product of the function $R(s, q)=$ $R_{s}(q)=s-q$ is $S^{-1}(s, q)$.

Proof. We have that $R_{s}^{c}(q)=\bar{s}-q$ and $R_{s}^{s}(q)=\left(q^{2}-2 \operatorname{Re}(s) q+|s|^{2}\right)$. 
We also have:

Proposition 2.17. The function $S^{-1}(s, q)$ is left regular in the variable $q$ and right regular in the variable $s$ in its domain of definition.

Proof. The regularity in $q$ follows by construction. The right regularity in $s$ follows by direct computations.

Remark 2.18. The right regular inverse of the function $R(s, q)=R_{q}(s)$ is the function ( $s-$ $\bar{q})\left(s^{2}-\operatorname{Re}(q) s+|q|^{2}\right)^{-1}$ (see remark 2.15) which, by identity (14), coincides with $S^{-1}(s, q)$.

Definition 2.19. Given a quaternion $q=x+I_{p} y\left(x, y \in \mathbb{R}\right.$ and $\left.I_{q} \in \mathbb{S}\right)$, we will denote by $\Sigma_{q}=x+\mathbb{S} y$ the 2 -sphere of its (generalized) conjugates.

Note that the 2 -sphere $\Sigma_{q}$ is equivalently defined by the conditions $|q|=x^{2}+y^{2}$ and $\operatorname{Re}(q)=x$.

Proposition 2.20. Let $q \in \mathbb{H} \backslash \mathbb{R}$. The singularities of the function $S^{-1}(s, q)=S_{q}^{-1}(s)=$ $-\left(q^{2}-\operatorname{Re}(s) q+|s|^{2}\right)^{-1}(q-\bar{s})$ lie on the 2-sphere $S_{q}$. More precisely: on the plane $L_{I}, I \neq I_{q}$ the restriction of the function $S^{-1}(s, q)$ to $L_{I}$ has the two singularities $\operatorname{Re}(q) \pm I|\operatorname{Im}(q)|$; while on the plane $L_{I_{q}}$ the restriction of the function $S^{-1}(s, q)$ to $L_{I_{q}}$ has the only singularity $q$. When $q \in \mathbb{R}$, then $S_{q}^{-1}(s)=(q-s)^{-1}$ and the only singularity is $q$.

Proof. Suppose $q \in \mathbb{H} \backslash \mathbb{R}$. The singularities of $S_{q}^{-1}(s)$ correspond to the roots of $|s|^{2}-2 \operatorname{Re}(s) q+$ $q^{2}=0$. This equation can be written by splitting real and imaginary parts as $|s|^{2}-2 \operatorname{Re}(s) \operatorname{Re}(q)+$ $\operatorname{Re}(q)^{2}-|\operatorname{Im}(q)|^{2}=0,(\operatorname{Re}(s)-\operatorname{Re}(q)) \operatorname{Im}(q)=0$. This implies $\operatorname{Re}(s)=\operatorname{Re}(q)$ and so $|s|=|q|$ i.e. the singularities consist of the whole 2 -sphere $\Sigma_{q}$. Consider the plane $L_{I}, I \neq I_{q}$ : it intersects the 2-sphere $\Sigma_{q}$ in $\operatorname{Re}(q) \pm I|\operatorname{Im}(q)|$. When $I=I_{q}$, or $q$ is real, then $q$ and $s$ commute, so $S_{q}^{-1}(s)=-(q-s)^{-1}(q-\bar{s})^{-1}(q-\bar{s})=(q-s)^{-1}$ and the statement follows.

\subsection{The Cauchy integral formula}

In this section we will present a new Cauchy formula (5), which holds for functions defined on a larger class of domains.

Lemma 2.21. Let $f, g$ be quaternionic valued, continuously (real) differentiable functions on an open set $U_{I}$ of the plane $L_{I}$. Then for every open $W \subset U_{I}$ whose boundary consists of a finite number of piecewise smooth, closed curves we have

$$
\int_{\partial W} g d s_{I} f=2 \int_{W}\left(\left(g \bar{\partial}_{I}\right) f+g\left(\bar{\partial}_{I} f\right)\right) d \sigma
$$

where $s=x+I y$ is the variable on $L_{I}, d s_{I}=-I d s$ and $d \sigma=d x \wedge d y$.

Proof. Let us choose an imaginary unit $J$ orthogonal to $I$ and let us consider $\mathbb{H}$ as the algebra generated by $I, J$. Then it is possible to write $f(s)=f_{0}(s)+f_{1}(s) J, g(s)=g_{0}(s)+J g_{1}(s)$ for suitable $L_{I}$-valued functions $f_{i}(s), g_{i}(s), i=0,1$. We now use the usual Stokes' theorem to write

$$
\begin{gathered}
\int_{\partial W} g d s_{I} f=\int_{\partial W}\left(g_{0}(s)+J g_{1}(s)\right) d s_{I}\left(f_{0}(s)+f_{1}(s) J\right) \\
=\int_{\partial W} g_{0} f_{0} d s_{I}+g_{0} f_{1} d s_{I} J+J g_{1} f_{0} d s_{I}+J g_{1} f_{1} d s_{I} J \\
=\int_{W} \partial_{x}\left(g_{0} f_{0}\right) d \sigma+\partial_{y}\left(g_{0} f_{0}\right) I d \sigma+\partial_{x}\left(g_{0} f_{1}\right) d \sigma J+\partial_{y}\left(g_{0} f_{1}\right) I d \sigma J+
\end{gathered}
$$




$$
+J \partial_{x}\left(g_{1} f_{0}\right) d \sigma+J \partial_{y}\left(g_{1} f_{0}\right) I d \sigma+J \partial_{x}\left(g_{1} f_{1}\right) d \sigma J+J \partial_{y}\left(g_{1} f_{1}\right) I d \sigma J .
$$

By direct computations we get

$$
\begin{gathered}
\partial_{x}\left(g_{0} f_{0}\right)+\partial_{y}\left(g_{0} f_{0}\right) I+J \partial_{x}\left(g_{1} f_{0}\right)+J \partial_{y}\left(g_{1} f_{0}\right) I \\
=\left(\partial_{x}\left(g_{0}\right)+\partial_{y}\left(g_{0}\right) I\right) f_{0}+J\left(\partial_{x}\left(g_{1}\right)+\partial_{y}\left(g_{1}\right) I\right) f_{0} \\
+g_{0}\left(\partial_{x}\left(f_{0}\right)+\partial_{y}\left(f_{0}\right) I\right)+J g_{1}\left(\partial_{x}\left(f_{0}\right)+\partial_{y}\left(f_{0}\right) I\right) \\
=\left(g_{0} \bar{\partial}_{I}+J g_{1} \bar{\partial}_{I}\right) f_{0} \\
+\left(g_{0}+J g_{1}\right)\left(\bar{\partial}_{I} f_{0}\right)=2\left(g \bar{\partial}_{I}\right) f_{0}+2 g\left(\bar{\partial}_{I} f_{0}\right)
\end{gathered}
$$

and analogously

$$
\partial_{x}\left(g_{0} f_{1}\right) J+\partial_{y}\left(g_{0} f_{1}\right) I J+J \partial_{x}\left(g_{1} f_{1}\right) J+J \partial_{y}\left(g_{1} f_{1}\right) I J=2\left(g \bar{\partial}_{I}\right) f_{1} J+2 g\left(\bar{\partial}_{I} f_{1}\right) J .
$$

Therefore we conclude

$$
\begin{gathered}
\int_{\partial W} g d s_{I} f=2 \int_{W}\left(g \bar{\partial}_{I}\right) f_{0} d \sigma+g\left(\bar{\partial}_{I} f_{0}\right) d \sigma+\left(g \bar{\partial}_{I}\right) f_{1} J d \sigma+g\left(\bar{\partial}_{I} f_{1}\right) J d \sigma \\
=2 \int_{W}\left(\left(g \bar{\partial}_{I}\right) f+g\left(\bar{\partial}_{I} f\right)\right) d \sigma
\end{gathered}
$$

An immediate consequence of the Lemma is the following:

Corollary 2.22. Let $f$ and $g$ be a left regular and a right regular function, respectively, on an open set $U \in \mathbb{H}$. For any $I \in \mathbb{S}$ and every open $W \subset U_{I}$ whose boundary consists of a finite number of piecewise smooth, closed curves, we have:

$$
\int_{\partial W} g d s_{I} f=0
$$

We will now identify a class of domains that naturally qualify as domains of definition of regular functions.

Definition 2.23. Let $\Omega \subseteq \mathbb{H}$ be a domain in $\mathbb{H}$. We say that $\Omega$ is a slice domain (s-domain for short) if $\Omega \cap \mathbb{R}$ is non empty and if $L_{I} \cap \Omega$ is a domain in $L_{I}$ for all $I \in \mathbb{S}$.

A regular function defined on a s-domain has a quaternionic series expansion which converges in a small open ball centered at a real point of the domain (see theorem 2.2), and hence it is very easy to prove that the identity principle stated in theorem 2.3 holds for regular functions defined on s-domains. For our purpose here we will also make use of the following

Definition 2.24. Let $\Omega \subseteq \mathbb{H}$. We say that $\Omega$ is circular if, for all $x+y I \in \Omega$, the whole 2-sphere $x+y \mathbb{S}$ is contained in $\Omega$.

We will focus our attention on the study of regular functions defined on circular s-domains. In fact these functions and domains turn out to be the natural setting of validity of the Cauchy formula that we are going to present. To construct this formula, we need now to extend to a wider class of regular functions the following value distribution property of regular quaternionic power series proved in [9]: 
Theorem 2.25. Let $f: B=B(0, R) \rightarrow \mathbb{H}$ defined by $f(q)=\sum_{n \geq 0} q^{n} a_{n}$ be a regular function. For all $x, y \in \mathbb{R}$ such that $x+y \mathbb{S} \subseteq B$ there exist $b, c \in \mathbb{H}$ such that

$$
f(x+y I)=b+I c
$$

for all $I \in \mathbb{S}$.

We will in fact prove here the following extension of theorem 2.25 (see [3] for the case of slice monogenic functions):

Theorem 2.26 (Representation Formula). Let $f$ be an regular function on a circular domain $\Omega \subseteq \mathbb{H}$. Choose any $J \in \mathbb{S}$. Then the following equality holds for all $q=x+y I \in \Omega$ :

$$
f(x+y I)=\frac{1}{2}[f(x+y J)+f(x-y J)]+I \frac{1}{2}[J[f(x-y J)-f(x+y J)]] .
$$

Moreover, for all $x, y \in \mathbb{R}$ such that $x+y \mathbb{S} \subseteq \Omega$, there exist $b, c \in \mathbb{H}$ such that for all $K \in \mathbb{S}$ we have

$$
\frac{1}{2}[f(x+y K)+f(x-y K)]=b \quad \text { and } \quad \frac{1}{2}[K[f(x-y K)-f(x+y K)]]=c .
$$

Proof. If $\operatorname{Im}(q)=0$ is real, the proof is immediate. Otherwise let us define the function $\psi: \Omega \rightarrow$ $\mathbb{H}$ as follows

$$
\begin{gathered}
\psi(q)=\frac{1}{2}[f(\operatorname{Re}(q)+|\operatorname{Im}(q)| J)+f(\operatorname{Re}(q)-|\operatorname{Im}(q)| J) \\
\left.+\frac{\operatorname{Im}(q)}{|\operatorname{Im}(q)|} J[f(\operatorname{Re}(q)-|\operatorname{Im}(q)| J)-f(\operatorname{Re}(q)+|\operatorname{Im}(q)| J)]\right] .
\end{gathered}
$$

Using the fact that $q=x+y I, x, y \in \mathbb{R}, y \geq 0$ and $I=\frac{\operatorname{Im}(q)}{|\operatorname{Im}(q)|}$ we obtain

$$
\psi(x+y I)=\frac{1}{2}[f(x+y J)+f(x-y J)+I J[f(x-y J)-f(x+y J)]] .
$$

Observe that on $L_{J}$ (i.e. for $I=J$ ) we have

$$
\psi_{J}(q)=\psi(x+y J)=f(x+y J)=f_{J}(q) .
$$

Therefore if we prove that $\psi$ is regular on $\Omega$, the first part of the assertion will follow from the Identity Principle for regular functions. Indeed, since $f$ is regular on $\Omega$, for any $I \in \mathbb{S}$ we have, on $\Omega \cap L_{I}$

$$
\begin{gathered}
\frac{\partial}{\partial x} 2 \psi(x+y I)=\frac{\partial}{\partial x}[f(x+y J)+f(x-y J)+I J[f(x-y J)-f(x+y J)]] \\
=\frac{\partial}{\partial x} f(x+y J)+\frac{\partial}{\partial x} f(x-y J)+I J\left[\frac{\partial}{\partial x} f(x-y J)-\frac{\partial}{\partial x} f(x+y J)\right] \\
=-J \frac{\partial}{\partial y} f(x+y J)+J \frac{\partial}{\partial y} f(x-y J)+I J\left[J \frac{\partial}{\partial y} f(x-y J)+J \frac{\partial}{\partial y} f(x+y J)\right] \\
=-J \frac{\partial}{\partial y} f(x+y J)+J \frac{\partial}{\partial y} f(x-y J)-I\left[\frac{\partial}{\partial y} f(x-y J)+\frac{\partial}{\partial y} f(x+y J)\right] \\
=-I \frac{\partial}{\partial y}[f(x+y J)+f(x-y J)+I J[f(x-y J)-f(x+y J)]]=-I \frac{\partial}{\partial y} 2 \psi(x+y I)
\end{gathered}
$$


i.e.

$$
\frac{1}{2}\left(\frac{\partial}{\partial x}+I \frac{\partial}{\partial y}\right) \psi(x+y I)=0 .
$$

To prove (17) we take any $K \in \mathbb{S}$ and use equation (16) to show that

$$
\begin{gathered}
\frac{1}{2}[f(x+y K)+f(x-y K)] \\
=\frac{1}{2}\left\{\frac{1}{2}[f(x+y J)+f(x-y J)]+K \frac{1}{2}[J[f(x-y J)-f(x+y J)]]\right. \\
\left.+\frac{1}{2}[f(x+y J)+f(x-y J)]-K \frac{1}{2}[J[f(x-y J)-f(x+y J)]]\right\} \\
=\frac{1}{2}[f(x+y J)+f(x-y J)]
\end{gathered}
$$

and that

$$
\begin{gathered}
\frac{1}{2}[K[f(x-y K)-f(x+y K)]] \\
=\frac{1}{2} K\left\{\frac{1}{2}[f(x+y J)+f(x-y J)]-K \frac{1}{2}[J[f(x-y J)-f(x+y J)]]\right. \\
\left.-\frac{1}{2}[f(x+y J)+f(x-y J)]-K \frac{1}{2}[J[f(x-y J)-f(x+y J)]]\right\} \\
=\frac{1}{2} K[-K[J[f(x-y J)-f(x+y J)]] \\
=\frac{1}{2}[J[f(x-y J)-f(x+y J)]] .
\end{gathered}
$$

With these two last equalities, the proof is completed.

The Representation Formula plays a key role in the study of the theory of regular functions on circular s-domains and we provide here some of its important consequences. Further developments will be the subject of a forthcoming paper.

Corollary 2.27. An regular function $f: \Omega \rightarrow \mathbb{H}$ on a circular s-domain is infinitely differentiable on $\Omega$.

Proof. The differentiability of $f$ on the real axis follows from Theorem 2.2 since for any point of the real axis there is a ball in which the function $f$ can be expressed in power series. To prove differentiability outside the real axis consider formula (16) in terms of $q=x_{0}+i x_{1}+j x_{2}+k x_{3}$, namely

$$
\begin{gathered}
f(q)=\frac{1}{2}[f(\operatorname{Re}(q)+|\operatorname{Im}(q)| J)+f(\operatorname{Re}(q)-|\operatorname{Im}(q)| J) \\
\left.+\frac{\operatorname{Im}(q)}{|\operatorname{Im}(q)|} J[f(\operatorname{Re}(q)-|\operatorname{Im}(q)| J)-f(\operatorname{Re}(q)+|\operatorname{Im}(q)| J)]\right] .
\end{gathered}
$$

Notice that the function $f$ is regular and hence infinitely differentiable on $L_{J}$. It is therefore obvious that $f$ can be obtained as a composition of the functions $f_{J}, \operatorname{Re}(q), \operatorname{Im}(q)$ and $|\operatorname{Im}(q)|$ which are all infinitely differentiable outside the real axis with respect to the variables $x_{\ell}$, $\ell=0, \ldots, 3$. This concludes the proof.

The following results are geometry-flavored consequences of the above theorem:

Corollary 2.28. Let $\Omega \in \mathbb{H}$ be a circular s-domain and let $f: \Omega \rightarrow \mathbb{H}$ be a regular function. 
1. For all $x, y \in \mathbb{R}$ such that $x+y I \in \Omega$ there exist $b, c \in \mathbb{H}$ such that

$$
f(x+y I)=b+I c
$$

for all $I \in \mathbb{S}$. In particular the image $f(x+y \mathbb{S})$ of the 2 -sphere $x+y \mathbb{S}$ is the 2 -sphere $b+\mathbb{S} c$.

2. If $f(x+y J)=f(x+y K)$ for $I \neq K$ in $\mathbb{S}$, then $f$ is constant on $x+y \mathbb{S}$; In particular, if $f(x+y J)=f(x+y K)=0$ for $I \neq K$ in $\mathbb{S}$, then $f$ vanishes on the entire 2 -sphere $x+y \mathbb{S}$.

Proof. The proof of 1 . is a direct application of theorem 2.26. To prove 2. notice that $f(x+y J)=$ $f(x+y K)$ for $I \neq K$ implies $c=0$ in (19), and hence the assertion follows.

We can now prove the new version of the Cauchy formula, which makes use of the class of circular s-domains naturally containing all the singularities of the regular kernel (for the case of slice monogenic functions see [3]).

Theorem 2.29. Let $\Omega \subseteq \mathbb{H}$ be a circular s-domain such that $\partial\left(\Omega \cap L_{I}\right)$ is union of a finite number of rectifiable Jordan arcs. Let $f$ be a regular function on $\Omega$ and, for any $I \in \mathbb{S}$, set $d s_{I}=-I d s$. Then for every $q \in \Omega$ we have:

$$
f(q)=\frac{1}{2 \pi} \int_{\partial\left(\Omega \cap L_{I}\right)}-\left(q^{2}-2 \operatorname{Re}(s) q+|s|^{2}\right)^{-1}(q-\bar{s}) d s_{I} f(s) .
$$

Moreover the value of the integral depends neither on $\Omega$ nor on the imaginary unit $I \in \mathbb{S}$.

Proof. First of all, the integral does not depend on the open set $\Omega$. This follows from the right regularity of $S^{-1}(s, q)$ with respect to the variable $s$ (see equality (14) and Corollary 2.22).

Let us show that the integral does not depend on the choice of the imaginary unit $I \in \mathbb{S}$. If we denote $q=x+y I_{q} \in \Omega$, then the set of the zeroes of the function $q^{2}-2 \operatorname{Re}(s) q+|s|^{2}=0$ consists of a real point (counted twice) or a 2-sphere. If the zeroes are not real, on any complex plane $L_{I}$ we find the two zeroes $s_{1,2}=x \pm y I$. When the singularity is a real number, the integral reduces to the classical Cauchy integral formula for holomorphic maps. Thus we consider the case of nonreal zeroes and we calculate the residues about the points $s_{1}$ e $s_{2}$. Let us start with $s_{1}=x+y I$ setting the positions

$$
\begin{gathered}
s=x+y I+\varepsilon e^{I \theta}, \\
\operatorname{Re}(s)=x+\varepsilon \cos \theta, \\
\bar{s}=x-y I+\varepsilon e^{-I \theta}, \\
d s_{I}=-I\left[\varepsilon I e^{I \theta}\right] d \theta=\varepsilon e^{I \theta} d \theta, \\
|s|^{2}=x^{2}+2 x \varepsilon \cos \theta+\varepsilon^{2}+y^{2}+2 y \varepsilon \sin \theta .
\end{gathered}
$$

We now compute the integral which appears at the right hand side of (20) along the circle with center at $s_{1}$ and radius $\varepsilon>0$ on the plane $L_{I}$ :

$$
\begin{gathered}
2 \pi I_{1}^{\varepsilon}=\int_{0}^{2 \pi}-\left(-2 q \varepsilon \cos \theta+2 x \varepsilon \cos \theta+\varepsilon^{2}+2 y \varepsilon \sin \theta\right)^{-1}\left(q-\left[x-y I+\varepsilon e^{-I \theta}\right]\right) \varepsilon e^{I \theta} d \theta f\left(x+y I+\varepsilon e^{I \theta}\right) \\
\quad=\int_{0}^{2 \pi}-(-2 q \cos \theta+2 x \cos \theta+\varepsilon+2 y \sin \theta)^{-1}\left(q-\left[x-y I+\varepsilon e^{-I \theta}\right]\right) e^{I \theta} d \theta f\left(x+y I+\varepsilon e^{I \theta}\right) .
\end{gathered}
$$


For $\varepsilon \rightarrow 0$ we get an expression $I_{1}^{0}$ for the residue at $s_{1}$

$$
\begin{gathered}
2 \pi I_{1}^{0}=\int_{0}^{2 \pi}(2 q \cos \theta-2 x \cos \theta-2 y \sin \theta)^{-1}\left(y I_{q}+y I\right) e^{I \theta} d \theta f(x+y I) \\
=\frac{1}{2} \int_{0}^{2 \pi}\left(y \cos \theta I_{q}-y \sin \theta\right)^{-1}\left(y I_{q}+y I\right) e^{I \theta} d \theta f(x+y I) \\
=-\frac{1}{2 y^{2}} \int_{0}^{2 \pi}\left(y \cos \theta I_{q}+y \sin \theta\right)\left(y I_{q}+y I\right)[\cos \theta+I \sin \theta] d \theta f(x+y I) \\
=-\frac{1}{2 y^{2}} \int_{0}^{2 \pi}\left[\left(y I_{q}\right)^{2} \cos \theta+y^{2} \sin \theta I_{q}+y^{2} \cos \theta I_{q} I+y^{2} \sin \theta I\right][\cos \theta+I \sin \theta] d \theta f(x+y I) \\
=-\frac{1}{2} \int_{0}^{2 \pi}\left[-\cos \theta+\sin \theta I_{q}+\cos \theta I_{q} I+\sin \theta I\right][\cos \theta+I \sin \theta] d \theta f(x+y I) \\
=-\frac{1}{2} \int_{0}^{2 \pi}\left[-\cos { }^{2} \theta-\cos \theta \sin \theta I+\cos \theta \sin \theta I_{q}+\sin ^{2} \theta I_{q} I+\cos ^{2} \theta I_{q} I\right. \\
\left.-\cos \theta \sin \theta I_{q}+\cos \theta \sin \theta I-\sin { }^{2} \theta\right] d \theta f(x+y I) \\
=-\frac{1}{2} \int_{0}^{2 \pi}\left[-1+I_{q} I\right] d \theta f(x+y I) \\
=\pi\left[1-I_{q} I\right] f(x+y I) .
\end{gathered}
$$

So we get the first residue

$$
I_{1}^{0}=\frac{1}{2}\left[1-I_{q} I\right] f(x+y I) .
$$

With analogous calculation we prove that the residue about $s_{2}$ is

$$
I_{2}^{0}=\frac{1}{2}\left[1+I_{q} I\right] f(x-y I)
$$

By the classical residues theorem used in the complex plane $L_{I}$, we have:

$$
\frac{1}{2 \pi} \int_{\partial\left(U \cap L_{I}\right)} S^{-1}(s, q) d s_{I} f(s)=I_{1}^{0}+I_{2}^{0}
$$

Since

$$
\begin{gathered}
I_{1}^{0}+I_{2}^{0}=\frac{1}{2}\left[1-I_{q} I\right] f(x+y I)+\frac{1}{2}\left[1+I_{q} I\right] f(x-y I) \\
=\frac{1}{2}[f(x+y I)+f(x-y I)]+I_{q} \frac{1}{2}[I[f(x-y I)-f(x+y I)]],
\end{gathered}
$$

the statement now follows from Lemma 2.26,

Corollary 2.30. Let $I \in \mathbb{S}$ and let $\Omega_{I}$ be a domain in $L_{I}$, symmetric with respect to the real axis and whose boundary is a finite union of closed Jordan arcs. Suppose that $\Omega_{I} \cap \mathbb{R}$ is nonempty. Let $J \in \mathbb{S}$ be orthogonal to $I$, let $F, G: \Omega_{I} \rightarrow L_{I}$ be holomorphic functions and let $f(x+y I)=F(x+y I)+G(x+y I) J . I f$

$$
\Omega=\bigcup_{x+y I \in \Omega_{I}}(x+y \mathbb{S})
$$


then the function defined by

$$
\tilde{f}(q)=\frac{1}{2 \pi} \int_{\partial \Omega_{I}}-\left(q^{2}-2 R e(s) q+|s|^{2}\right)^{-1}(q-\bar{s}) d s_{I} f(s)
$$

is the regular extension of $f$ to $\Omega$.

Proposition 2.31 (Derivatives using the regular Cauchy kernel). Let $U \subset \mathbb{H}$ be a circular sdomain. Suppose $\partial\left(U \cap L_{I}\right)$ is a finite union of rectifiable Jordan curves for every $I \in \mathbb{S}$. Let $f$ be an regular function on $U$ and set $d s_{I}=d s / I$. Let $q, s$. Then

$$
\begin{gathered}
\partial_{x}^{n} f(q)=\frac{n !}{2 \pi} \int_{\partial\left(U \cap L_{I}\right)}\left(q^{2}-2 s_{0} q+|s|^{2}\right)^{-n-1}(q-\bar{s})^{*(n+1)} d s_{I} f(s) \\
=\frac{n !}{2 \pi} \int_{\partial\left(U \cap L_{I}\right)}\left[S^{-1}(s, q)(q-\bar{s})^{-1}\right]^{n+1}(q-\bar{s})^{*(n+1)} d s_{I} f(s)
\end{gathered}
$$

where

$$
(q-\bar{s})^{* n}=\sum_{k=0}^{n} \frac{n !}{(n-k) ! k !} q^{n-k} \bar{s}^{k},
$$

is the $n$-th power with respect to the *-product. Moreover, the integral does not depend on $U$ and on the imaginary unit $I \in \mathbb{S}$.

Proof. First of all, we recall that the derivative coincides, for regular functions, with the partial derivative with respect to the scalar coordinate $x=\operatorname{Re}(q)$. Therefore, we can identify $\partial_{x}^{n} f(q)=$ $\partial_{s}^{n} f(q)$. To compute $\partial_{x}^{n} f(q)$, it is enough to compute the derivative of the integrand, since $f$ and its derivatives with respect to $x$ are continuous functions on $\partial\left(U \cap L_{I}\right)$. Thus we get

$$
\partial_{x}^{n} f(q)=\frac{1}{2 \pi} \int_{\partial\left(U \cap L_{I}\right)} \partial_{s}^{n}\left[S^{-1}(s, q)\right] d s_{I} f(s) .
$$

To prove the statement, it is sufficient to compute $\partial_{x}^{n}\left[S^{-1}(s, q)\right]$. We proceed by recurrence. Consider the derivative $\partial_{x} S^{-1}(s, q)$ :

$$
\begin{gathered}
\partial_{x} S^{-1}(s, q)=-\left(q^{2}-2 \operatorname{Re}(s) q+|s|^{2}\right)^{-2}(2 q-2 \operatorname{Re}(s))(q-\bar{s})-\left(q^{2}-2 \operatorname{Re}(s) q+|s|^{2}\right)^{-1} \\
=\left(q^{2}-2 \operatorname{Re}(s) q+|s|^{2}\right)^{-2}\left[2 q^{2}-2 q \bar{s}-2 \operatorname{Re}(s) q+2 \operatorname{Re}(s) \bar{s}-q^{2}+2 \operatorname{Re}(s) q-|s|^{2}\right] \\
=\left(q^{2}-2 \operatorname{Re}(s) q+|s|^{2}\right)^{-2}\left[q^{2}-2 q \bar{s}+\bar{s}^{2}\right]=\left(q^{2}-2 \operatorname{Re}(s) q+|s|^{2}\right)^{-2}(x-\bar{s})^{* 2} .
\end{gathered}
$$

We now assume

$$
\partial_{x}^{n} S^{-1}(s, q)=(-1)^{n+1} n !\left(q^{2}-2 \operatorname{Re}(s) q+|s|^{2}\right)^{-(n+1)}(q-\bar{s})^{*(n+1)},
$$

and we compute $\partial_{x}^{n+1} S^{-1}(s, q)$. We have:

$$
\begin{gathered}
\partial_{x}^{n+1} S^{-1}(s, q)=\partial_{x}\left[(-1)^{n+1} n !\left(q^{2}-2 \operatorname{Re}(s) q+|s|^{2}\right)^{-(n+1)}(q-\bar{s})^{*(n+1)}\right] \\
=(-1)^{n+2}(n+1) !\left(q^{2}-2 \operatorname{Re}(s) q+|s|^{2}\right)^{-(n+2)}(2 q-2 \operatorname{Re}(s))(q-\bar{s})^{*(n+1)} \\
+(-1)^{n+1}(n+1) !\left(q^{2}-2 \operatorname{Re}(s) q+|s|^{2}\right)^{-(n+1)}(q-\bar{s})^{* n} \\
=(-1)^{n+2}(n+1) !\left(q^{2}-2 \operatorname{Re}(s) q+|s|^{2}\right)^{-(n+2)} \\
\times\left[(2 q-2 \operatorname{Re}(s))(q-\bar{s})-\left(q^{2}-2 \operatorname{Re}(s) q+|s|^{2}\right)\right] *(q-\bar{s})^{* n},
\end{gathered}
$$


where we have used the fact that the regular product coincides with the usual one when the coefficients a real numbers. Therefore

$$
\begin{gathered}
\partial_{x}^{n+1} S^{-1}(s, x)=(-1)^{n+2}(n+1) ! \\
\times\left(q^{2}-2 \operatorname{Re}(s) q+|s|^{2}\right)^{-(n+2)}\left[q^{2}-2 q \bar{s}+\bar{s}^{2}\right] *(q-\bar{s})^{* n} .
\end{gathered}
$$

The last equality in (21) depends on the fact that $S^{-1}(s, q)(q-\bar{s})^{-1}=\left(q^{2}-2 \operatorname{Re}(s) q+|s|^{2}\right)^{-1}$.

Remark 2.32. The proposition above provides an alternative way to prove that, on circular s-domains, a regular function $f$ is infinitely differentiable, see Corollary 2.27.

Provided the importance of the regular Cauchy kernel that we have introduced, we conclude the paper by presenting two different explicit series expansions.

Theorem 2.33. Let $q$ and $s=u+v I(I \in \mathbb{S}, v>0)$ be two quaternions such that

$$
v<|q-u|
$$

Then the noncommutative Cauchy kernel can be represented by the series

$$
S^{-1}(s, q)=\sum_{n \geq 0}(q-u)^{-n-1}(v I)^{n}
$$

Proof. Consider the equalities

$$
\begin{gathered}
\left(q^{2}-2 q u+u^{2}+v^{2}\right)^{-1} \\
=\left((q-u)^{2}+v^{2}\right)^{-1} \\
=\left((q-u)^{2}\left(1+v^{2}(q-u)^{-2}\right)\right)^{-1} \\
=\left(1+v^{2}(q-u)^{-2}\right)^{-1}(q-u)^{-2} \\
=\sum_{n \geq 0}(-1)^{n} v^{2 n}(q-u)^{-2 n-2} .
\end{gathered}
$$

We now multiply the last expression by $(q-u+v I)$ on the right hand side and obtain:

$$
\begin{gathered}
S^{-1}(s, q)=\sum_{n \geq 0}(-1)^{n} v^{2 n}(q-u)^{-2 n-2}(q-u+v I) \\
=\sum_{n \geq 0}(-1)^{n} v^{2 n}(q-u)^{-2 n-1}+\sum_{n \geq 0}(-1)^{n} v^{2 n}(q-u)^{-2 n-2} v I
\end{gathered}
$$

Since $(-1)^{n} v^{2 n}=(v I)^{2 n}$ is a real number we obtain

$$
=\sum_{n \geq 0}(q-u)^{-2 n-1}(v I)^{2 n}+\sum_{n \geq 0}(q-u)^{-2 n-2}(v I)^{2 n+1}
$$

from which the statement follows.

To conclude, we now examine what happens on the complement of the closure of the domain in which the series above converges. We will adopt a Laurent type approach: 
Theorem 2.34. Let $q$ and $s=u+v I(I \in \mathbb{S}, v>0)$ be two quaternions such that

$$
|q-u|<v
$$

Then the S-resolvent operator admits the series expansion:

$$
S^{-1}(s, q)=\sum_{n \geq 0}(q-u)^{n}(v I)^{-n-1} .
$$

Proof. We have the following equalities:

$$
\begin{gathered}
\left(q^{2}-2 q u+u^{2}+v^{2}\right)^{-1}=\left[(q-u)^{2}+v^{2}\right]^{-1}=v^{-2}\left[1+(q-u)^{2} v^{-2}\right]^{-1} \\
=v^{-2}\left[\sum_{n \geq 0}(-1)^{n}(q-u)^{2 n} v^{-2 n}\right] .
\end{gathered}
$$

By multiplying the last equality by $(q-u+v I)$ on the right hand side, we obtain:

$$
S^{-1}(s, q)=\sum_{n \geq 0}(-1)^{n}(q-u)^{2 n+1} v^{-2 n-2}+\sum_{n \geq 0}(-1)^{n}(q-u)^{2 n} v^{-2 n-2}(v I) .
$$

Recalling that $(-1)^{n} v^{2 n}=(v I)^{2 n}$ is a real number we get:

$$
S^{-1}(s, q)=\sum_{n \geq 0}-(q-u)^{2 n+1}(v I)^{-2 n-2}+\sum_{n \geq 0}-(q-u)^{2 n}(v I)^{-2 n-1}
$$

which is our statement. The series converges when (25) holds.

\section{References}

[1] F. Colombo, G. Gentili, I. Sabadini, D.C. Struppa, A functional calculus in a non commutative setting, Electron. Res. Announc. Math. Sci, 14 (2007), 60-68.

[2] F. Colombo, G. Gentili, I. Sabadini, D.C. Struppa, Non commutative functional calculus: bounded operators, preprint, 2007.

[3] F. Colombo, I. Sabadini, The Cauchy formula with s-monogenic kernel and a functional calculus for noncommuting operators, preprint, 2008.

[4] F. Colombo, I. Sabadini, D.C. Struppa, Slice monogenic functions, to appear in Israel Journal of Mathematics.

[5] F. Colombo, I. Sabadini, D.C. Struppa, A new functional calculus for noncommuting operators, J. Funct. Anal., 254 (2008), 2255-2274.

[6] F. Colombo, I. Sabadini, F. Sommen, D.C. Struppa, Analysis of Dirac Systems and Computational Algebra, Progress in Mathematical Physics, Vol. 39, Birkhäuser, Boston, 2004.

[7] R. Fueter, Die Funktionentheorie der Differentialgleichungen $\triangle u=0$ und $\triangle \triangle u=0$ mit vier reellen Variablen, Comm. Math. Helv. 7 (1934), 307-330.

[8] R. Fueter, Über eine Hartogs'schen Satz, Comment. Math. Helv., 12 (1939/40), 75-80. 
[9] G. Gentili, C. Stoppato, Zeros of regular functions and polynomials of a quaternionic variable, to appear in Michigan Math. J.

[10] G. Gentili, C. Stoppato, The open mapping theorem for quaternionic regular functions, E-print arXiv:0802.3861 v1 [math.CV].

[11] G. Gentili, D.C. Struppa, A new approach to Cullen-regular functions of a quaternionic variable, C.R. Acad. Sci. Paris, 342 (2006), 741-744.

[12] G. Gentili, D.C. Struppa, A new theory of regular functions of a quaternionic variable, Adv. Math. 216 (2007), 279-301.

[13] G. Gentili, D.C. Struppa, Regular functions on a Clifford Algebra, Complex Var. Elliptic Equ. 53 (2008), 475-483.

[14] T.Y. Lam, A first course in noncommutative rings. Graduate Texts in Mathematics, 123. Springer-Verlag, New York, 1991, 261-263.

[15] A. Pogorui, M.V. Shapiro, On the structure of the set of zeros of quaternionic polynomials, Complex Variables 49, (2004) no. 6, 379-389.

[16] R. Serôdio, L.-S. Siu, Zeros of quaternion polynomials. Appl. Math. Letters 14 (2001), 237-239. 